\title{
Use of Donor Serum to Prevent Passive Transfer of Hyperacute Rejection
}

\author{
Jue Wang, M.D., Edward K. Geissler, Ph.D., John H. Fechner, JR., M.S., \\ William J. Burlingham, Ph.D., and Stuart J. KNechtle, M.D. \\ Department of Surgery, University of Wisconsin, Madison, Wisconsin 53792
}

Presented at the Annual Meeting of the Association for Academic Surgery, Hershey, Pennsylvania, November 10-13, 1993

Organ transplantation in presensitized recipients continues to be contraindicated for heart and kidney recipients due to the risk of hyperacute rejection, which has no known treatment at this time. We tested whether donor serum, which contains soluble MHC class I antigen, is able to neutralize the effect of antidonor antibody in the recipient and prevent hyperacute or accelerated rejection. A rat model of passive immunization was used to test the role of anti-donor antibody in hyperacute rejection. Seven of 10 recipients of hyperimmune serum (HyS), derived from Lewis rats (RT1') following 3 ACI (RT1") skin grafts, developed hy peracute or accelerated rejection. Intravenous injection of $\mathrm{ACI}$ serum prior to the HyS administration prevented hyperacute rejection in all recipients tested. When third-party (Wistar-Furth, R'T1") serum was given to Lewis rats injected with $\mathrm{HyS}$, hyperacute rejection was not abrogated. When examining the mechanism of this effect, a simple antibody blocking phenomenon was found to be unlikely since flow cytometry analysis showed that ACI serum needed to be present at $\geqslant 256$-fold excess compared to HyS to block anti-ACI antibody binding to RT1. $A^{*}+$ cells by $50 \%$. We tested whether the RT1.A* class I antigen in ACI serum had other biologic properties that resulted in the prolonged graft survival. However, removal of RT1.A antigen from $\mathrm{ACl}$ serum prior to use in the passive transfer model did not abrogate the graft prolongation observed previously. These data suggest that components of donor serum other than MHC class I antigen may be useful for preventing the antibody-mediated component of hy peracute rejection. 1991 Acndemic Prena, Ine.

\section{INTRODUCTION}

Renal transplantation in presensitized patients with preformed anti-donor antibody is contraindicated due to the risk of hyperacute or accelerated rejection. No current immunosuppressive modalities are effective in preventing antibody-mediated damage. If a cross-match test prior to human renal transplantation is positive, a

$0022-4804 / 94 \$ 5.00$

Copyright 1994 by Academic Press, Inc.

All rights of reproduction in any form regerved. different organ recipient must be selected. Currently $40 \%$ of the patients awaiting renal transplantation are highly sensitized ( $>40 \%$ panel-reactive antibody) to potential donors, substantially reducing their chances of receiving a renal transplant. New solutions to immunotherapy in sensitized patients are urgently needed.

The purpose of this study was to determine whether soluble MHC class I antigen of the donor type has the ability to prevent or at least downregulate the immunologic injury caused by anti-donor antibody. We therefore chose a rat model in which passive transfer of $\mathrm{HyS}$ at the time of cardiac allografting causes hyperacute rejection. Donor serum was used as a source of soluble class I antigen. The reason for using a passive transfer model was to exclude the contribution of the primed cellular immune response.

\section{MATERIALS AND METHODS}

\section{Animals}

Inbred male $\mathrm{ACI}$ rats ( $\mathrm{R}^{\prime} \mathrm{T}^{\mathrm{a}}{ }^{\mathrm{a}}$ ) were donors for skin and cardiac allografts. Male Lewis rats (RT1') were recipients for all procedures, and Wistar-Furth (WF) rats $\left(\mathrm{RT} 1^{\circ}\right)$ were used as third-party controls. All rats weighed between 150 and $200 \mathrm{~g}$ and were obtained from Harlan Sprague-Dawley (Indianapolis, IN).

\section{HyS Preparation}

Two-centimeter-diameter ACI skin grafts were grafted onto the dorsal neck area of Lewis rats. After the first skin graft was rejected, the second and third ACI skin grafts were subsequently placed at 10 - to 14 -day intervals. HyS was collected 14-20 days after the third skin graft rejected. Sera from groups of sensitized Lewis rats were combined to make batches of pooled $\mathrm{HyS}$. Batches of $\mathrm{HyS}$ were stored at $-20^{\circ} \mathrm{C}$ for later use.

Detection of Anti-RT1.A Antibody and RT'1.A Antigen

Serum samples were diluted in phosphate-buffered saline containing $0.5 \%$ bovine serum albumin. Fifty mi- 


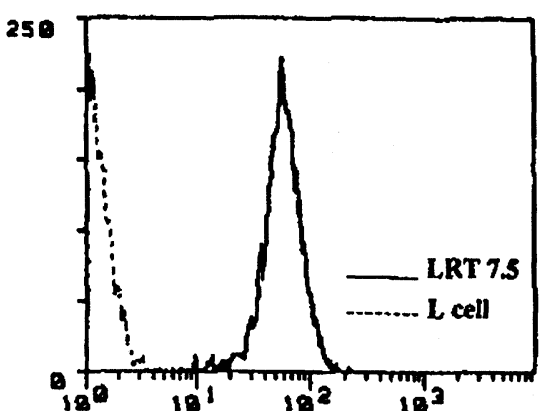

FIG. 1. Flow cytometry histogram showing expression of RT1.A in mouse $\mathrm{L}$ cells stably transfected with the cDNA for RT1.A* Nontransfected $L$ cells reacted with the same RT1. $A^{*}$-specific antibody are shown. To demonstrate the expression of RT1. $A^{*}$, cells were first reacted with $\mathrm{R} 3 / 13$ antibody (Bioproducts for Science, Indianapolis, IN) and then with goat anti-rat IgG-FITC

croliters of diluted serum was reacted with $5 \times 10^{5}$ LRT7.5 cells in a $12 \times 75-\mathrm{mm}$ polystyrene tube for 30 min at $4^{\circ} \mathrm{C}$. LRT7.5 cells are a clone derived from mouse L-cells that were stably transfected with the cDNA encoding RT1.A" (cDNA provided by Dr. Jonathan Howard, Babraham Institute, Cambridge, UK); these cells constitutively express the RT1. $A^{\mathrm{a}}$ molecule on their surface (Fig. 1). After a 30-min incubation of the cell/serum mixture, cells were washed with diluent and resuspended to $100 \mu \mathrm{l}$. Two microliters of goat anti-rat IgG-FITC (Jackson ImmunoResearch) was added to each tube and reacted for $30 \mathrm{~min}$ at $4^{\circ} \mathrm{C}$. Cells were washed and analyzed by flow cytometry.

In some experiments, sera were combined to determine whether soluble RT1.A in ACI serum could neutralize anti-RT1. $\mathrm{A}^{\mathrm{a}}$ antibodies in HyS. HyS and either $\mathrm{ACI}$ or normal Lewis (control) serum were mixed at various ratios, and incubated $1 \mathrm{hr}$ at room temperature.
After incubation, $50 \mu \mathrm{l}$ of each serum mixture was combined with $5 \times 10^{5}$ LRT7.5 cells and left at $4^{\circ} \mathrm{C}$ for 30 min. Cells were washed, reacted with goat anti-rat IgGFITC as described above, and analyzed by flow cytometry. The mean channel of fluorescence (MCF) was determined with the control reaction at each ratio of normal Lewis serum to HyS. The percent maximum MCF was calculated by dividing the MCF determined at a given ratio of ACI serum to HyS by the MCF determined at the same ratio of normal Lewis serum to HyS (control reaction).

The level of RT1.A antigen present in ACI serum was tested using a specific ELISA which has been described in detail previously [1]. Purified RT1.A [1] was used as a standard to determine the RT1. $A^{\mathbf{a}}$ concentration.

\section{Depletion of RT1. $A^{a}$ from ACI Serum}

RT1.A $A^{a}$ was depleted from ACI serum using Sepharose 4B (Sigma, St. Louis, MO) coupled to both OX-18 (specific for monomorphic determinant of RT1.A; Bioproducts for Science, Indianapolis, IN) and RT1.A*-specific monoclonal antibody 211-4D9 (hybridoma provided by Heinz Kunz, University of Pittsburgh, Pittsburgh, PA). Serum was passed through the column $3 \times$ and was tested for RT1.A before and after the depletion procedure.

\section{Cardiac Transplantation, Serum Transfer, and Histology}

Heterotopic abdominal cardiac allografting was performed by the modified microvascular technique as described previously [2]. Cardiac allografts were checked every $2 \mathrm{hr}$ for the first $24 \mathrm{hr}$ and then daily. The time of rejection was defined by palpation and direct visual inspection at laparotomy. Animals were bled via the tail vein to obtain serum for antibody testing. Serum was passively transferred intravenously following ACI heart

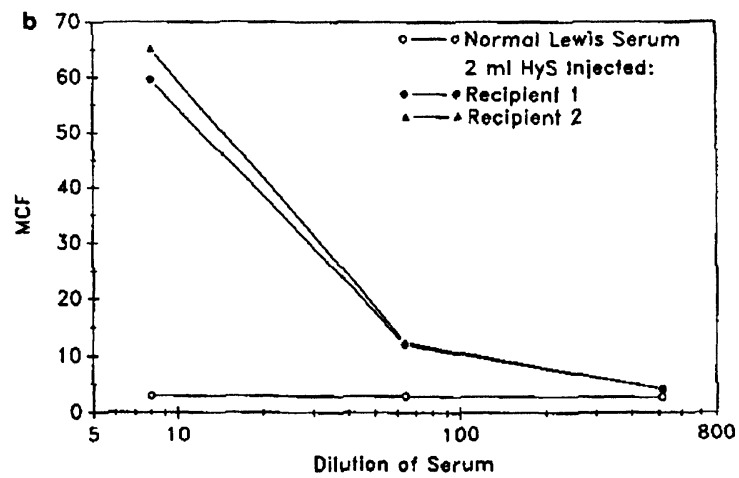

FIG. 2. (a) Measurement of anti-RTI.A* antibody titer in batches of HyS. Batches of HyS were diluted and tested for reactivity with the RT1.A*-expressing clone, LRT7.5. Antibody binding to LRT7.5 cells was detected with goat anti-rat IgG-FITC and was measured by determining the mean channel of fluorescence (MCF) using flow cytometry. The titers of different batches of HyS are shown. (b) Measurement of anti-RT1.A4 antibody titer after $\mathrm{HyS}$ serum injection into Lewis rats. Serum was obtained $1 \mathrm{hr}$ after injection of HyS into two Lewis rat heart allograft recipients. The titers of anti-RT1. $A^{*}$ antibody in these sera are shown. 

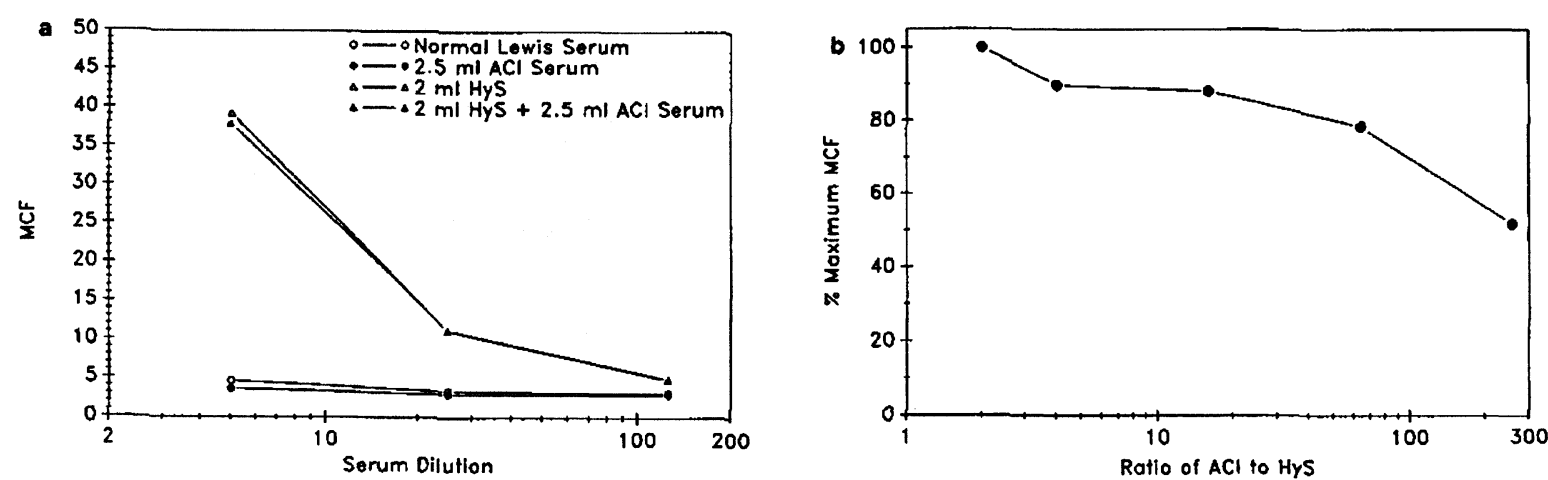

FIG. 3. (a) Effect of ACI serum injection on anti-RT1.A antibody titer in an allograft recipient injected with HyS. The titer of anti-RT1.A antibody in an allograft recipient was determined by flow cytometry $1 \mathrm{hr}$ after injection of an allograft recipient with only $\mathrm{HyS}$ and $1 \mathrm{hr}$ after

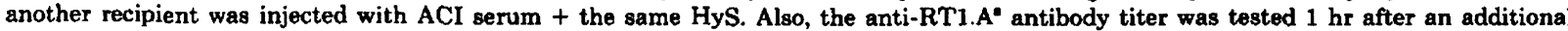
recipient was injected with only $\mathrm{ACl}$ serum. (b) The in vitro effect of $\mathrm{ACl}$ serum addition on the amount of anti-RTl. $\mathrm{A}^{*}$ antibody in $\mathrm{HyS}$. ACl serum was combined with $\mathrm{HyS}$ at various ratios, and $60 \mu \mathrm{l}$ of each serum mixture was reacted with LRT7.5 cells to determine anti-RT1.A antibody levels. The MCF at each ratio was compared to the maximum MCF, as determined by reacting normal Lewis aerum with HyS at the same ratio. The \% maximum MCF at each ratio of ACI to HyS is shown.

transplantation. Animals in each of five groups were injected with: (1) $2.0 \mathrm{ml} \mathrm{HyS}$, (2) $2.5 \mathrm{ml} \mathrm{ACI}$ serum plus 2.0 $\mathrm{ml} \mathrm{HyS}$, (3) $2.5 \mathrm{ml} \mathrm{WF}$ serum plus $2.0 \mathrm{ml} \mathrm{HyS}$, (4) $2.5 \mathrm{ml}$ ACI serum, or (5) no serum.

Rejected hearts were formalin-fixed and embedded in paraffin. Six - to 8- $\mu \mathrm{m}$ sections were cut and stained with hematoxylin and eosin.

\section{RESULTS}

Pooled HyS was tested for the presence of antiRT1.A antibody. Flow cytometric analysis of HyS showed consistently high titers of anti-RT1.A $A^{\mathbf{a}}$ antibody in the different batches of HyS (Fig. 2a). Recipient serum obtained $1 \mathrm{hr}$ after both heart transplantation and injection of $2.0 \mathrm{ml} \mathrm{HyS}$, showed high levels of antidonor antibody (Fig. 2b).

TABLE 1

Donor Serum Prevents Passive Transfer of Hyperacute Rejection

\begin{tabular}{cccccc}
\hline & & ACI & WF & $\begin{array}{c}\text { Graft survival time } \\
\text { (days) }\end{array}$ & $P$ value \\
\hline Group & HyS & serum & serum & \\
\hline 1. & - & - & - & $6,6,6,6,7$ & \\
2. & - & + & - & $4,6,6,6,6,7$ & NS vs 1 \\
3. & + & - & - & $0.2,0.2,0.9,1.1,2$, & 0.05 vs 1 \\
& & & & $2,4,8,8,8$ & \\
4. & + & + & - & $8,9,9,9,9,9,10$, & 0.004 vs 3 \\
& & & & 10 & \\
5. & + & - & + & $0.6,0.9,2,2,8,8,9$ & NS vs 3 \\
6. & + & 6 & - & $9,10,10,10,10$ & NS vs 4 \\
\hline
\end{tabular}

Note. NS, not significant $(P>0.10)$.

a Mann-Whitney test.

${ }^{b}+\mathrm{ACI}$ serum depleted of RT1.A $\mathrm{A}^{a}$ antigen.
The effect of ACI serum injection on the titer of antiRT1. $A^{*}$ antibody in HyS-injected heart allograft recipients was determined. Transplanted rats injected with HyS serum, as well as ACI serum, did not show a decrease in anti-RT1. $A^{\mathrm{a}}$ antibody levels compared to transplanted rats receiving only HyS (Fig. 3a). Serum collected at the same time point in transplanted rats injected with only ACI serum did not have detectable anti-RT1.A antibody present. To test whether the soluble RT1. $A^{a}$ in ACI serum is able to effectively reduce the titer of anti-RT1. $\mathrm{A}^{\mathrm{a}}$ antibody in $\mathrm{HyS}$ in vitro, ACI and HyS were mixed at various ratios, and the antibody titer was subsequently determined by flow cytometric analysis (Fig. 3b). To reduce the MCF exhibited with HyS on LRT7.5 cells by $50 \%, A C I$ serum needed to be combined with $\mathrm{HyS}$ at a 256:1 ratio, respectively. Lower ratios of $\mathrm{ACI}$ to HyS serum reduced the MCF by $\leqslant 20 \%$. To ensure the presence of RT1.A" antigen in ACI serum, an RT1.A'-specific ELISA was performed. Soluble RT1.A* was detectable at a concentration of $195 \pm 92$ (SD) $\mathrm{ng}$ / $\mathrm{ml}$ in normal ACI serum $(n=5)$.

Table 1 summarizes the survival times of heterotopic cardiac allografts in naive Lewis rats or rats injected with various combinations of sera. In the control group where no serum injections were performed, ACI hearts rejected at 6-7 days. Injection of $2.5 \mathrm{ml} \mathrm{ACI} \mathrm{serum} \mathrm{alone}$ had no effect on rejection time. Transplanted rats injected with only HyS showed hyperacute or accelerated rejection in 7 of 10 recipients. Administration of ACI serum, prior to HyS, resulted in prolonged graft survival compared to HyS alone in all eight animals tested. To test the specificity of the effect of ACI serum, WF (third-party) serum was injected along with $\mathrm{HyS}$ after heart transplantation. The results show that four of seven grafts were rejected in a hyperacute or accelerated manner. Histologic evaluation of hearts rejected early 

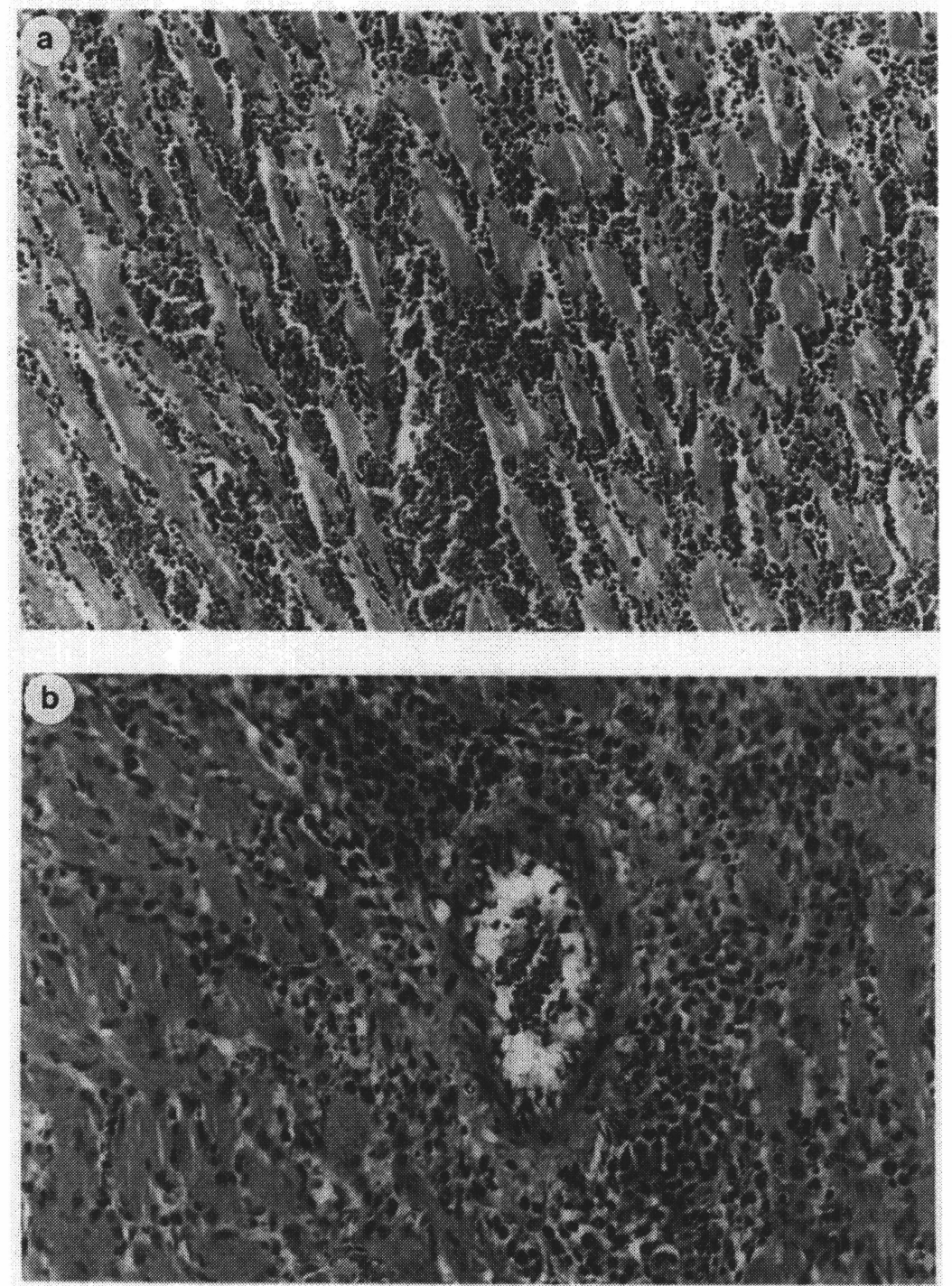

FIG. 4. (a) Cardiac allografts with hyperacute rejection developed severe edema, hemorrhage, infarction, and polymorphonuclear cell infiltration. (b) Hearts that rejected acutely ( $\geqslant 6$ days) showed massive lymphocyte infiltration and myocyte necrosis.

( $<4$ days), regardless of treatment, showed features typical of humoral rejection with severe tissue edema, hemorrhage, infarction, and polymorphonuclear cell infiltration (Fig. 4a). In contrast, hearts rejected at time points $>7$ days showed histological evidence of severe lymphocytic infiltration, endothelialitis, and necrosis of myocytes with replacement by fibroblasts (Fig. 4 b).

To determine whether soluble MHC class I antigen in donor-type serum has the ability to prevent or downregulate the immunologic injury caused by anti-donor antibody, ACI serum was depleted of RT1.A antigen by affinity chromatography (Fig. 5) prior to injection with HyS. Data from these experiments show that the re- moval of RT1.A by this method did not abrogate the ability of ACI serum to prolong graft survival in the passive transfer model (Table 1).

\section{DISCUSSION}

Hyperacute rejection of transplanted organs is believed to be mediated primarily by donor-specific antibody present in the recipient at the time of transplantation. Donor-specific antibody binds to donor histocompatibility antigens, principally MHC class I antigen within the allograft, and initiates a complement-dependent form of rapidly progressive tissue destruction [3-5]. 


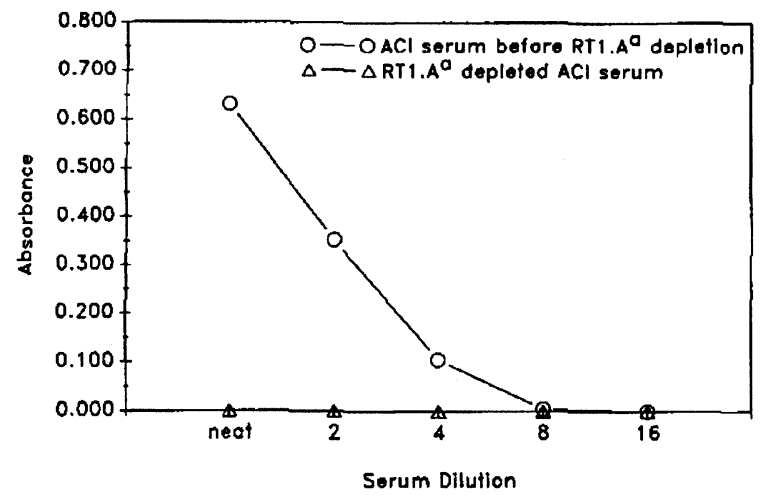

FIG. 5. Depletion of RT1.A* from ACI serum. ACI serum was depleted of RT1.A* by affinity chromatography and was subsequently tested for RT1.A* in an ELISA. Absorbance levels at the various serum dilutions are shown above.

It has previously been demonstrated that hyperacute rejection of rat hepatic allografts can be readily produced in recipients presensitized by donor skin when the appropriate inbred strain combinations are used [6]. The ACI to Lewis rat strain combination is a high-responder strain combination. In high-responder models, when three successive skin grafts are performed on the recipient, hyperacute rejection of skin, heart, and liver consistently occurs both in an actively and a passively immunized recipient $[4,7,8]$. It has also been shown that immediately following liver transplantation the liver releases significant amounts of soluble MHC class I molecules from the donor liver $[9,10]$. Infusion of soluble class $I$ antigen derived from liver extracts has been shown to prolong survival of subsequent cardiac allografts in a donor-specific manner in a nonsensitized model [11]. One explanation of the liver's relative resistance to hyperacute rejection compared to other vascularized organ allografts is that the donor-type MHC class I antigen blocks the destructive effects of antidonor antibody, either by neutralizing the antibody or through immunomodulatory effects of immune complexes formed between the donor type MHC class I antigen and donor-specific antibody [11-13]. However, a precise role of donor MHC class I antigen in conferring resistance to antibody-mediated rejection remains unclear. The goal of the current investigation was to isolate the effects of donor-type MHC class I antigen on antibody-mediated rejection of cardiac allografts using donor serum as a source of MHC class I antigen. The contribution of the sensitized cellular immune response was removed by passively transferring hyperimmune serum to an immunologically naive recipient.

In the present study, hyperacute rejection was prevented in a passive transfer model by injection of donor serum. The inhibition of hyperacute rejection by donor serum occurred despite the fact that the titer of anti- donor antibody was unchanged in vivo with injection of ACI serum. Additionally, when $\mathrm{HyS}$ and ACI serum were combined in vitro, ACI serum needed to be added in large excess to reduce the anti-RT1. $A^{a}$ antibody titer substantially. Therefore, donor serum used in this man. ner is not a feasible approach for lowering antibody titers, and our results cannot be explained by a simple antibody-blocking phenomenon.

Donor serum was shown to contain substantial amounts of donor-type MHC class I antigen. The inability of third-party serum to prevent hyperacute rejection suggested that the soluble antigens in the donor serum, principally MHC antigens, may have been responsible for this effect. These results are consistent with the hypothesis that soluble donor MHC class I antigen can inactivate or downregulate antibody-mediated rejection. However, when RT1.A* class I antigen was depleted from ACI serum, the effect on antibody-mediated rejection in the passive transfer model was not abrogated. These results suggest that class I antigen is not the component of donor serum preventing hyperacute rejection, or RT1.A* depletion from ACI serum was not complete. Incomplete depletion of RT1.A is a possibility since the ELISA is only sensitive to $10 \mathrm{ng} / \mathrm{ml}$. Another explanation is that MHC antigens other than RT1.A (Lewis and ACI strains are a complete MHC mismatch) are involved in this effect.

The prolongation of graft survival in recipients of hyperimmune serum who do not hyperacutely reject their cardiac allografts could be due to the well-described phenomenon of antiidiotypic antibody production which prevents acute rejection temporarily [14-16]. Why some recipients of HyS undergo hyperacute rejection while others have prolonged survival with passive enhancement is unclear. The possibility of inducing hyperacute rejection by administering hyperimmune serum makes this strategy too risky as a means of preventing acute cellular rejection.

The remarkable resistance of the liver to antibodymediated rejection $[17,18]$ may in part be due to its release of soluble $\mathrm{MHC}$ antigens following transplantation. Although our study suggests that serum MHC class I antigen alone plus anti-donor antibody is not solely responsible for preventing hyperacute or accelerated rejection in a passive transfer model, the mechanism of the donor-serum effect in this model warrants further investigation using more concentrated sources of MHC antigen. Further studies using recombinant MHC antigens are in progress to test its utility in preventing hyperacute or accelerated rejection in sensitized models of organ transplantation.

\section{REFERENCES}

1. Geissler, E. K., Wang, J., Fechner, J. H., Jr., Burlingham, W. J., and Knechtle, S. J. Immunity to MHC class I antigen following 
direct DNA transfer into skeletal muscle. J. Immunol. 152: 413, 1994.

2. Ono, K., and Lindsey, E. S. Improved technique of heart transplantation in rats. J. Thorac. Cardiovasc. Surg. 57: 391, 1969.

3. Caves, P. K., Dong, E., Jr., Morris, R. E., and Shumway, N. E. Hyperacute rejection of orthotopic cardiac allografts in dogs following solubilized antigen pretreatment. Transplantation 16: $252,1973$.

4. Knechtle, S. J., Halperin, E. C., Murphy, C. E., Saad, T., Abernethy, K., Miller, D., and Bollinger, R. R. The effect of cyclosporine, total lymphoid irradiation, and cobra venom factor on hyperacute rejection. Heart Transplant. 4: 541, 1985.

5. Forbes, R. D. C., Guttmann, R. D., and Pinto-Blonde, M. A passive transfer model of hyperacute rat cardiac allograft rejection. Lab. Invest. 41: 348, 1979.

6. Knechtle, S. J., Yamaguchi, Y., Coundouriotis, A., and Bollinger, R. R. Mediation of hyperacute rejection of rat hepatic allografts by RT-1 antigens. Transplantation 48: 723, 1989 .

7. Guttmann, R. D. Genetics of acute rejection of rat cardiac allografts and a model of hyperacute rejection. Transplantation 17: $383,1974$.

8. Knechtle, S. J., Kolbeck, P. C., Tsuchimoto, S., Coundouriotis, A., Sanfilippo, F., and Bollinger, R. R. Humoral rejection of rat hepatic transplants by passive transfer of serum. Transplant. Proc. 19: 1072, 1987.

9. Davies, H. ff. S., Pollard, S. G., and Calne, R. Y. Soluble HLA antigens in the circulation of liver graft recipients. Transplantation 47: 524, 1989.

10. Kamada, N., and Wight, D. G. D. Antigen-specific immunosuppression induced by liver transplantation in the rat. Transplantation 38: 217, 1984.
11. Sumimoto, R., and Kamada, N. Specific suppression of allograft rejection by soluble class I antigen and complexes with monoclonal antibody. Transplantation 50: 678, 1990.

12. Kamada, $\mathrm{N}$. The immunology of experimental liver transplantation in the rat. Immunology 55: 369, 1985.

13. Kamada, N., Shinomiya, T., Tamaki, T., and Ishiguro, K. Immunosuppressive activity of serum from liver grafted rats: Passive enhancement of fully allogeneic heart grafts and induction of systemic tolerance. Transplantation 42: 581, 1986.

14. Mohanakumar, T., Rhodes, C., Mendez-Picon, G., Flye, M. W., and Lee, H. M. Antidiotypic antibodies to human major histocompatibility complex class I and II antibodies in hepatic transplantation and their role in allograft survival. Transplantation 44: 54, 1987.

15. Suciu-Foca, N., Reed, E., D'Agati, V. D., Ho, E., Cohen, D. J., Benvenisty, A. I., McCabe, R., Brensilver, J. M., King, D. W., and Hardy, M. A. Soluble HLA antigens, anti-HLA antibodies, and antiidiotypic antibodies in the circulation of renal transplant recipients. Transplantation 61: 593, 1991.

16. Wasfie, T., Reed, E., Suciu-Foca, N., and Hardy, M. A. Production of antiidiotypic antibodies in the rat: In vitro characteriza. tion of specificity and correlation with in vivo specific suppression of cardiac allograft immune reaction across major histocom. patability complex. Surgery 108: 431, 1990.

17. Fung, J. J., Makowka, L., Griffin, M., Duquesnoy, R. J., Tsakis, A., and Starzl, T. E. Successful sequential liver-kidney transplantation in patients with preformed lymphocytotoxic antibodies. Clin. Transplant. 1: 187, 1987.

18. Iwatsuki, S., Iwaki, Y., Kano, T., Klintmalm, G., Koep, L. J., Weil, R., and Starzl, T. E. Successful liver transplantation from crossmatch-positive donors. Transplant. Proc. 13: 286, 1981. 